\title{
O IMPACTO DO BASQUETEBOL EM CADEIRA DE RODAS NO NÍVEL DE COLESTEROL-HDL DE MULHERES COM LESÃO MEDULAR
}

\author{
THE IMPACT OF WHEELCHAIR BASKETBALL ON HDL-CHOLESTEROL LEVELS IN \\ WOMEN WITH SPINAL CORD INJURY
}

\begin{abstract}
RESUMO
O propósito do presente estudo foi objetivo avaliar o impacto da prática de basquetebol em cadeira de rodas sobre o perfil lipídico de pacientes paraplégicos do sexo feminino. Foram avaliados os níveis séricos da HDL-C, da LDL-C e do colesterol total (CT) de dois grupos atendidos num programa de reabilitação. Um grupo foi formado por pacientes praticantes de basquetebol em CR (grupo PR), ( $n=4)$ e o outro por suas congêneres paraplégicas não praticantes (NPR), $(n=8)$. Todas as pacientes foram acometidas por LM. Duas coletas foram realizadas para cada grupo. O intervalo médio entre as coletas foi de 11 meses para o grupo PR $(n=4)$ e de 8 meses para o grupo NPR ( $=8)$. A atividade do grupo PR teve duração semanal média de 130 minutos. O teste t de Student foi utilizado para avaliar as variáveis. Um valor de $p \leq$ 0,05 foi considerado significante. Os valores iniciais do HDL-C e das relações CT/ HDL-C e LDL-C/HDL-C entre os grupos PR e NPR não apresentaram diferenças significativas $(p>0,05)$. Na segunda medição, comparando os grupos PR e NPR, os níveis séricos de HDL-C do grupo PR mostraram-se significativamente mais elevados $(p \leq 0,01)$. Pode-se concluir que a atividade semanal de basquetebol em cadeira de rodas foi eficaz na melhora do perfil lipídico das pacientes paraplégicas e podem reduzir o risco de doença cardiovascular. Esta melhora pode ter sido mediada pela adoção de um maior nível de atividade física diária por parte das praticantes.
\end{abstract}

Palavras-chave: Paraplegia; Atividade motora; Reabilitação; Colesterol.

\begin{abstract}
The objective of this study was to Assess the impact of wheelchair basketball (WB) on the lipid profile of female paraplegic patients. High-density lipoprotein cholesterol (HDL-C), low-density lipoprotein cholesterol (LDL-C) and total cholesterol (TC) serum levels from two groups were assessed. Both groups were enrolled in a rehabilitation program (RP). One group comprised patients who played WB (P group), $(n=4)$ and another group was made up of patients who did not play (NP), $(n=8)$. All patients enrolled had spinal cord injuries (SCl). Two blood samples were performed for each group. The mean interval between these samples was 11 months in the P group and 8 months to the NP group. The mean weekly duration of activity in $\mathrm{P}$ group was 130 minutes. Student's $t$ test and linear regression methods were used for statistical analysis. A p-value $\leq 0.05$ was considered statistically significant. The HDL-C, TC/HDL-C and LDL-C/HDL-C baseline values exhibited no significant differences between the $P$ and NP groups ( $p>0.05)$. In the second sample, after taking part in WB, comparing the two measurements, the mean HDL-C level in group P had increased significantly $(p \leq 0.01)$, by $27.42 \%$. It was conclude that weekly WB activity was effective for improving the lipid profile test results in paraplegic patients and may reduce cardiovascular disease risk. This improvement could have been mediated by the adoption of a higher level of daily physical activity by those exercising.
\end{abstract}

Key words: Paraplegia; Motor activity; Rehabilitation; Cholesterol.

1 Universidade Federal do Rio Grande do Norte (UFRN), Centro de Ciências da Saúde; Programa de Pós-Graduação em Ciências da Saúde 


\section{INTRODUÇÃO}

Um indivíduo com LM pode sofrer seqüelas físicas incapacitantes, culminando com importantes repercussões emocionais e na rede social dos indivíduos acometidos ${ }^{1}$. Apesar do avanço nos tratamentos das complicações advindas da LM e do aprimoramento dos programas de reabilitação que têm proporcionado uma maior expectativa de vida a estas pessoas, ao se comparar com a população geral, o índice de mortalidade nesta população ainda é consideravelmente alto ${ }^{2}$.

Existem evidências obtidas através de avaliação ergométrica, de uma maior prevalência de doenças cardiovasculares assintomáticas nesta população ${ }^{3}$. A doença cardiovascular, juntamente com os problemas respiratórios representam, atualmente, a maior afecção fatal nestes indivíduos ${ }^{4}$.

Estudos epidemiológicos conduzidos em grandes populações vêm confirmando que a HDL-C é o maior preditor independente de doença cardiovascular na população geral e que suas concentrações diminuídas aumentam os fatores de risco para doença cardiovascular, existindo uma correlação inversa entre os níveis séricos da HDL-C e doença arteriocoronariana ${ }^{5,6}$.

A HDL-C tem na sua composição uma maior parte protéica (mais de $50 \%$ da sua massa) em relação às outras lipoproteínas. Ela é sintetizada no fígado e no intestino e age captando o colesterol livre e levandoo para o fígado. Esta ação depuradora, associada a outros mecanismos, é considerada anti-aterogênica ${ }^{7,8}$. São também muito importantes as análises da LDL-C (lipoproteína de baixa intensidade), do colesterol total (CT) e da relação CT /HDL-C.

Embora a prática de exercícios físicos apropriados por parte de pessoas com LM seja extremamente importante para a saúde física, mental e social, favorecendo a independência e a reabilitação destas, no Brasil, poucos clubes, academias e centros esportivos encontram-se adaptados a receber estas pessoas com dificuldades de locomoção ${ }^{9}$. Estima-se que a maioria dos indivíduos com LM apresente um estilo de vida bastante sedentário ${ }^{10}$. Esta pode ser a razão pela qual, comumente, estes apresentem um perfil lipídico indesejável, colocando-os em situação de alto risco para doença cardiovascular ${ }^{11,21,13}$.

Estudando a população geral (sem lesão medular), uma meta-análise de 66 estudos evidenciou que a atividade física regular exerce efeito notável no perfil lipídico: diminuições no CT e na LDL-C e aumento na HDL-C. Algumas variáveis como valor inicial pré-treinamento, idade e tempo de treinamento podem interagir com as modificações positivas no perfil lipídico ${ }^{14}$. De fato, a maioria dos estudos com a população geral tem mostrado alterações benéficas no perfil lipídico decorrentes da prática de exercício físico regular aeróbico (o efeito agudo de uma sessão também têm sido observado) exercido a diferentes intensidades de esforço, freqüência e duração por diferentes grupos de pessoas de ambos os sexos, de diferentes faixas etárias, aptidões iniciais, dislipidêmicos e normolipidêmicos ${ }^{15}$. Uma revisão mais atual ${ }^{16}$ confirma alguns destes resultados, destacando a importância da atividade física aeróbia, embora um número ainda pequeno de estudos evidenciem bons resultados através dos exercícios resistidos. A contribuição da variável volume de treinamento parece ser mais importante do que a intensidade de treinamento, embora a última induza uma melhor aptidão física. Os níveis da HDL-C parecem estar mais fortemente associados ao volume de treinamento e os níveis de LDL-C e CT mais associados à restrição calórica, perda de peso e variações na composição corporal $^{16}$. Contudo, a prescrição do exercício é um campo que precisa ser melhor explorado.

O mecanismo responsável pela elevação dos níveis da HDL-C ainda não foi totalmente estabelecido, mas parece que o exercício físico estimula, através de um processo multi-fatorial, um aumento da atividade da lipoproteína lipase, da lecitina-colesterol-aciltransferase e por uma diminuição da lipase hepática ${ }^{8}$

Estas mudanças nos níveis da HDL-C, e na consequente análise do perfil lipídico, mediada pela atividade física, parecem se estender à população com $\mathrm{LM}^{17}$. Sendo assim, a atividade física regular tem se constituído uma importante ferramenta para elevar os níveis de HDL-C e reverter esta condição de alto risco para doença cardiovascular. Entretanto, muito pouco se sabe quanto ao tipo de exercício e a melhor maneira de recomendá-lo ${ }^{18}$, nesta população. O objetivo principal deste trabalho foi investigar o efeito da prática semanal do basquetebol em cadeira de rodas (BCR) no perfil lipídico de mulheres paraplégicas, com atenção especial à HDL-C e às relações colesterol total (CT) /HDL-C e lipoproteína de baixa densidade colesterol (LDL-C)/HDL-C.

\section{PROCEDIMENTOS METODOLÓGICOS}

O programa de reabilitação do Centro de Reabilitação Sarah-Fortaleza (CRSF) atende, além de outras patologias, pacientes com LM traumáticas e não traumáticas de uma grande região do nordeste brasileiro, incluindo os estados do Ceará, Rio Grande do Norte, Piauí, Pernambuco e Paraíba. O programa de reabilitação é composto por uma equipe multidisciplinar, na qual as atividades de educação física, na forma de orientações e vivências, integram um amplo conjunto de propostas oferecidas.

A prática do $\mathrm{BCR}$, sob a supervisão do profissional de educação física, é realizada semanalmente na quadra poliesportiva da instituição e conta com uma freqüência média de 17 pacientes de ambos os sexos, que participam voluntariamente do programa de basquetebol detalhado na Tabela I.

É importante esclarecer que já existe uma rotina de controle e acompanhamento das pacientes após o período de internação no programa de reabilitação através de avaliações sanguíneas, incluindo dosagem de lipoproteínas e triglicérides. As pacientes com idade superior a trinta anos recebem acompanhamento periódico anual da instituição. As mulheres com idade 
inferior a trinta anos que venham a apresentar níveis de colesterol fora do preconizado ${ }^{20}$ na admissão, também recebem acompanhamento. O conhecimento obtido acerca da problemática do perfil lipídico de pessoas com LM, aliado a esta rotina pré-existente, viabilizaram este estudo de caráter retrospectivo.

Tabela 1. Distribuição da prática do BCR.

\begin{tabular}{|c|c|}
\hline $\begin{array}{l}\text { Atividades realizadas durante a prática } \\
\text { de BCR }\end{array}$ & Tempo (minutos) \\
\hline $\begin{array}{l}\text { Exercícios de flexibilidade para o tronco } \\
\text { e membros superiores. }\end{array}$ & 10 \\
\hline $\begin{array}{l}\text { Exercícios de treinamento das capaci- } \\
\text { dades físicas: velocidade, resistência } \\
\text { aeróbia, potência e destrezas com a } \\
\text { CR (giros, mudanças de direção, arran- } \\
\text { ques, etc.). }\end{array}$ & 20 \\
\hline $\begin{array}{l}\text { Treinamento técnico-tático específico da } \\
\text { modalidade: recepção, controle de bola, } \\
\text { condução de bola com a CR, passes, ar- } \\
\text { remessos, pequenos jogos e simulações } \\
\text { de situações de jogo. }\end{array}$ & 55 \\
\hline $\begin{array}{l}\text { Descanso inclusive para ingestão } \\
\text { líquida }\end{array}$ & 10 \\
\hline $\begin{array}{l}\text { Ação coletiva de basquetebol por } \\
\text { equipe. }\end{array}$ & 30 \\
\hline $\begin{array}{l}\text { Relaxamento (exercícios de flexibilidade, } \\
\text { de respiração ou comentários sobre a } \\
\text { sessão de treino). }\end{array}$ & 05 \\
\hline Total & 130 \\
\hline
\end{tabular}

Através de pesquisa em prontuário médico informatizado, todas as pacientes paraplégicas do sexo feminino com LM, admitidas no programa de reabilitação $(n=55)$, em um período exato de dois anos, foram identificadas. grupos:

Dentre estas pacientes, foram identificados dois

a) o grupo das praticantes (PR) de BCR formado por sete pacientes. Destas, foram descartadas três pacientes por não reunirem no mínimo três meses na prática da atividade, tempo estimado necessário para se esperar algum efeito crônico do exercício físico sobre as lipoproteínas ${ }^{19}$.

b) o grupo controle foi formado por todas as pacientes paraplégicas por LM, admitidas neste período, não praticantes (NPR) do $B C R$, que haviam realizado ao menos dois exames de perfil lipídico neste período. Baseado nestes critérios, oito pacientes foram selecionadas.

Nenhuma paciente dos grupos PR e NPR, referiu hábito compatível com etilismo ou tabagismo e não seguiam dieta para controle de dislipidemia. As pacientes do grupo PR não praticavam nenhum tipo de atividade física recente antes de começarem a participar do BCR. Não obtivemos esta informação em relação ao grupo NPR.

Baseados na prática da nossa equipe de reabilitação e no conhecimento científico no que tange ao tratamento de complicações advindas da LM, constatamos que são freqüentes o uso de medicações anti-espásticas, anti-colinérgicos (para aumentar a capacidade vesical), medicações para dores neuropáticas, anti-depressivos, antibióticos (para combater episódios de infecção urinária), mas não existem relações entre estas medicações e o perfil lipídico'.

\section{A análise laboratorial}

Os níveis de triglicérides (TG) e CT foram analisados pelo método enzimático-COD-ANA e a HDL-C por determinação direta, através do aparelho EXPRESS PLUS-BAYER ${ }^{\circledR}$, da própria instituição. A LDL-C foi obtida pela equação de Friedwald, desde que o nível de TG não superasse $400 \mathrm{mg} / \mathrm{dL}$. LDL-C = CT - $\{\mathrm{HDL}-\mathrm{C}+(\mathrm{TG} / 5)\}$.

Os perfis lipídicos (com enfoque no HDL-C e nas suas relações) do grupo PR pré e pós participação na atividade foram comparados, assim como os perfis lipídicos do grupo NPR. O intervalo médio entre as coletas do grupo PR foi de 11 meses e o do grupo NPR foi oito meses.

\section{A análise estatística}

Os dados foram apresentados em valores absolutos e média \pm desvio padrão. Foi utilizado o programa SPSS Windows 13.0 para análise estatística dos dados. Primeiramente, uma regressão linear descartou a possível influência das variáveis idade, tempo de lesão e tempo transcorrido entre os exames na evolução dos resultados. Na comparação das variáveis HDL-C, CT/HD-C e LDL-C/HDL-C entre os grupos PR e NPR, na primeira e na segunda coleta, foi utilizado um teste $t$ de Student. Na avaliação da evolução das variáveis intra-grupo foi utilizado um teste $t$ de Student para dados pareados. Foi adotado como critério de significância o valor de $p \leq 0,05$. Apesar do tamanho relativamente pequeno da amostra, optamos por um teste paramétrico, já que as variáveis CT, LDL-C e HDL-C estão distribuídas normalmente, com variância igual e independência na observação.

\section{Aspectos éticos}

O Comitê de Ética e Pesquisa envolvendo Seres Humanos da instituição responsável pelo programa de reabilitação, constituído por integrantes locais e de sua sede e tendo como referência a resolução 196/96 do Conselho Nacional de Saúde, aprovou o uso das informações dos exames laboratoriais contidas em prontuário médico (Protocolo enviado ao CONEP $\mathrm{n}^{\circ}$ 21). Antes da participação no BCR, a adequação desta atividade, para cada indivíduo, é discutida pela equipe de reabilitação, baseada em exames físicos e no rigor profissional. Os riscos e benefícios desta atividade, para os pacientes selecionados, são previamente discutidos.

\section{RESULTADOS}

As características dos dois grupos estão descritas na Tabela 2. A média de idade do grupo PR foi de 49,8 \pm 
3,7 anos e o tempo de lesão de $9,2 \pm 5,6$ anos. A média de idade das pacientes do grupo NPR foi de 40,4 $\pm 5,7$ anos e do tempo de lesão $5,9 \pm 2,8$ anos. Os valores obtidos nas dosagens séricas do perfil lipoprotéico de cada paciente se encontram na tabela 3

A média de idade do grupo PR foi de 49,8 $\pm 3,7$ anos e o tempo de lesão de 9,2 $\pm 5,6$ anos (Tabela II). A média de idade das pacientes do grupo NPR foi de $40,4 \pm 5,7$ anos e do tempo de lesão 5,9 $\pm 2,8$ anos (Tabela 2). Os valores obtidos nas dosagens séricas do perfil lipoprotéico de cada paciente se encontram na tabela 3.

No grupo $\mathrm{PR}$, os valores obtidos na primeira dosagem da HDL-C variaram de 38 a $51 \mathrm{mg} / \mathrm{dL}$, a relação CT/HDL-C de 3,98 a 5,93 e a relação LDL-C/ HDL-C de 2,70 a 4,21. Na segunda avaliação da HDL-C observou-se variação de 53 a $60 \mathrm{mg} / \mathrm{dL}$, a relação CT/ HDL-C variou de 3,35 a 4,85 e a relação LDL-C/HD-C de 2,17 a 3,35 (Tabela III). Houve um aumento médio de $27,42 \%$ da HDL-C e uma diminuição de $16,34 \%$ e $18,78 \%$ nas relações CT/HDL-C e LDL-C/HDL-C respectivamente. Ocorreram diferenças significativas $(p \leq 0,05)$ entre a primeira e a segunda dosagem da HDL-C (fig 2) e também entre as relações CT/HDL-C $(p \leq 0,05)$, (fig. 3) e LDL-C/HDL-C ( $p \leq 0,05)$, (fig. 4).

No grupo NPR, os valores obtidos na primeira dosagem da HDL-C variaram de 31 a $61 \mathrm{mg} / \mathrm{dL}$, a relação CT/HDL-C de 3,11 a 6,30 e a relação LDLC/HDL-C de 2,08 a 4,51. Na segunda avaliação, a $\mathrm{HDL}-\mathrm{C}$ variou de 36 a $59 \mathrm{mg} / \mathrm{dL}$, a relação $\mathrm{CT} / \mathrm{HDL}-\mathrm{C}$ de 3,15 a 6,30 e a relação LDL/HDL-C de 1,77 a 4,49 (Tabela III). Houve uma diminuição média de $7,67 \%$ da HDL-C, um aumento de $2,46 \%$ na relação CT/HDL-C e uma diminuição de $3,13 \%$ na relação LDL/HDL, sem diferença estatística significativa.

Os valores iniciais da HDL-C (Figura 1) e das relações CT/HDL-C (Figura 2) e LDL-C/HDL-C (Figura 3) entre os grupos PR e NPR não apresentaram diferenças significativas $(p>0,05)$. O grupo PR apresentou elevação significativa (Figura 1) da HDL-C $(p \leq 0,01)$ e nenhuma alteração significativa $(p>0,05)$ das frações CT/HDL (Figura 2) e LDL/HDL (figura 3) em relação ao grupo NPR na segunda avaliação, apesar da tendência de queda nestas duas variáveis.

Tabela 2. Características das paraplégicas dos grupos PR e NPR.

\begin{tabular}{|c|c|c|c|c|}
\hline $\begin{array}{l}\text { CARACTERÍSTICAS DOS } \\
\text { GRUPOS }\end{array}$ & CAUSA DA LESÃO & NÍVEL DE LESÃO & $\begin{array}{l}\text { IDADE } \\
\text { (ANOS) }\end{array}$ & $\begin{array}{l}\text { TEMPO DE LESÃO } \\
\text { (ANOS) }\end{array}$ \\
\hline \multicolumn{5}{|l|}{$\overline{P R}$} \\
\hline 1 & Acidente Automobilistico & T2 & 54 & 11 \\
\hline 2 & Acidente Automobilistico & T4 & 45 & 7 \\
\hline 3 & Acidente Automobilistico & T6 & 50 & 16 \\
\hline 4 & Projétil de Arma de Fogo & T12 & 50 & 3 \\
\hline \multicolumn{5}{|l|}{ NPR } \\
\hline 1 & Acidente Automobilistico & T12 & 38 & 3 \\
\hline 2 & Projétil de Arma de Fogo & T7 & 44 & 9 \\
\hline 3 & Acidente Automobilistico & T4 & 45 & 5 \\
\hline 4 & Queda de Altura & T5 & 36 & 10 \\
\hline 5 & Acidente de Moto & T8 & 44 & 4 \\
\hline 6 & Queda de Altura & T11 & 37 & 2 \\
\hline 7 & Acidente Automobilistico & T3 & 48 & 7 \\
\hline 8 & Projétil de Arma de Fogo & T1 & 31 & 7 \\
\hline
\end{tabular}

PR $(n=4)$ = praticante de basquetebol em CR ; NPR $(n=8)$ = não praticante de basquetebol em CR; T = Torácico

Tabela 3. Valores das dosagens séricas do HDL-C e das relações CT/HDL-C e LDL/HDL-C por paciente.

\begin{tabular}{|c|c|c|c|c|c|c|}
\hline \multirow[t]{3}{*}{ GRUPOS/PACIENTES } & \multicolumn{6}{|c|}{ PERFIL LIPOPROTEICO } \\
\hline & \multicolumn{2}{|c|}{$\mathrm{HDL}(\mathrm{mg} / \mathrm{dL})$} & \multicolumn{2}{|c|}{$\mathrm{CT} / \mathrm{HDL}-\mathrm{C}$} & \multicolumn{2}{|c|}{ LDL-C/HDL-C } \\
\hline & $1^{\circ}$ & $2^{\circ}$ & $1^{\circ}$ & $2^{\circ}$ & $1^{\circ}$ & $2^{\circ}$ \\
\hline \multicolumn{7}{|l|}{ PR } \\
\hline 1 & 43 & 60 & 3,98 & 3,35 & 2,70 & 2,17 \\
\hline 2 & 38 & 55 & 5,16 & 4,04 & 3,42 & 2,58 \\
\hline 3 & 51 & 53 & 4,57 & 4,19 & 2,96 & 2,70 \\
\hline 4 & 43 & 55 & 5,93 & 4,85 & 4,21 & 3,35 \\
\hline \multicolumn{7}{|l|}{ NPR } \\
\hline 1 & 52 & 43 & 4,00 & 3,49 & 2,29 & 1,77 \\
\hline 2 & 49 & 39 & 5,29 & 6,28 & 3,80 & 4,49 \\
\hline 3 & 46 & 37 & 4,91 & 6,30 & 3,48 & 4,32 \\
\hline 4 & 45 & 36 & 4,38 & 5,14 & 2,13 & 2,36 \\
\hline 5 & 43 & 45 & 6,30 & 5,71 & 4,51 & 3,53 \\
\hline 6 & 61 & 59 & 3,11 & 3,15 & 2,08 & 1,93 \\
\hline 7 & 31 & 39 & 5,52 & 4,97 & 3,81 & 3,51 \\
\hline 8 & 38 & 39 & 3,82 & 3,21 & 2,53 & 1,95 \\
\hline
\end{tabular}




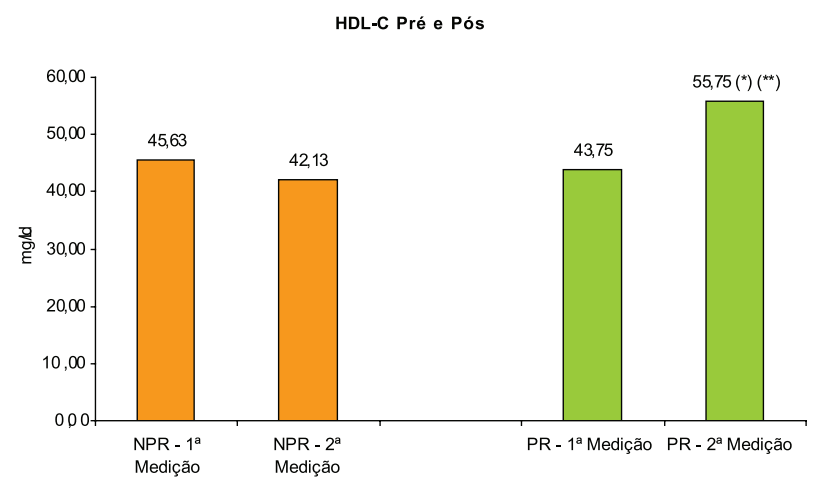

Figura 1. Valores da HDL-C das pacientes com $L M$, pré e pós BCR.

Valores expressos como média \pm desvio padrão.

${ }^{*} \leq 0,05 P R 1^{a}$ medição vs PR $2^{a}$ medição

${ }^{* *} p \leq 0,01$ PR $2^{a}$ medição vs NPR $2^{a}$ medição

CT/HDL-C Pré e Pós

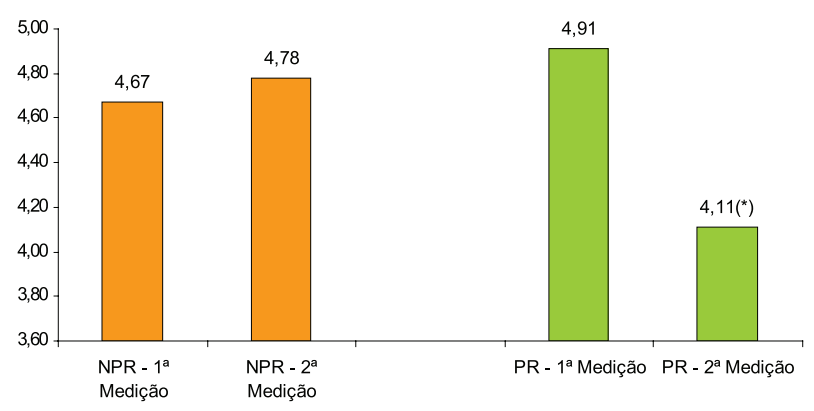

Figura 2. Valores da relação CT/HDL-C das pacientes com LM.

Valores expressos como média \pm desvio padrão.

* $\leq$ 0,05 PR $1^{a}$ medição vs $P R 2^{a}$ medição $p>0,05$ NPR $1^{a}$ medição vs NPR $2^{a}$ medição e

NPR $2^{a}$ medição vs PR $2^{a}$ medição

Os valores iniciais da HDL-C (Figura 1) e das relações CT/HDL-C (Figura 2) e LDL-C/HDL-C (Figura 3) entre os grupos PR e NPR não apresentaram diferenças significativas $(p>0,05)$. O grupo $P R$ apresentou elevação significativa (Figura 1) do HDL-C $(p \leq 0,01)$ e nenhuma alteração significativa $(p>0,05)$ das frações CT/HDL (Figura 2) e LDL/HDL (figura 3) em relação ao grupo NPR na segunda avaliação, apesar da tendência de queda nestas duas variáveis.

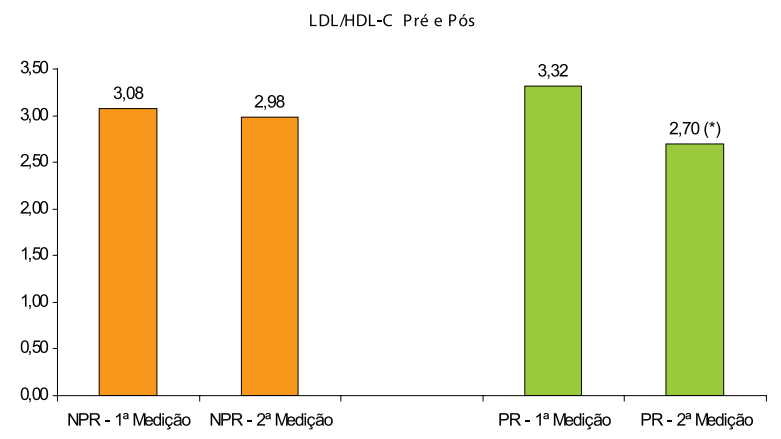

Figura 3. Valores da relação LDL-C/HDL-C das pacientes com LM.

Valores expressos como média \pm desvio padrão. * $\leq 0,05$ $P R 1^{a}$ medição vs $P R 2^{a}$ medição

\section{DISCUSSÃO}

Os valores da HDL-C obtidos neste estudo na população com LM são compatíveis com os valores obtidos em outros estudos realizados em outros países ${ }^{12}$. Parece pouco provável que fatores culturais ou mesmo ligados à etnia possam produzir resultados diferentes, embora um estudo tenha levantado esta possibilidade ${ }^{11}$ Ainda assim, estudos com populações maiores e regionalizados podem trazer mais conhecimento sobre os níveis da HDL-C nos pacientes com LM no Brasil.

Sabe-se que existem diferenças no perfil lipídico entre homens e mulheres pré-menopausa ${ }^{21}$. As mulheres normalmente apresentam valores da HDL-C acima dos valores obtidos em homens. Esta é uma diferença atribuída ao sexo (especificamente à produção do estrogênio). Os valores acima de 55mg/ dL são considerados aceitáveis segundo referência nacional ${ }^{20}$. O grupo PR tinha em sua maioria mulheres pós menopáusicas com a média de idade mais avançada em relação ao grupo NPR (49,8 vs 40,4 anos). Contudo, não existiam diferenças significativas entre os grupos nas variáveis analisadas na primeira medição.

Antes de participarem da atividade de BCR, as mulheres do grupo PR apresentavam níveis médios da HDL-C (43,75 mg/dL) que representavam um risco aumentado por volta de $42 \%$ para um ataque cardíaco em relação à população geral, de acordo as predições desenvolvidas no estudo de Framingham ${ }^{6}$, que considera esta variável, o melhor preditor independente de doença arteriocoronariana. Após o período médio de 11 meses de atividade de basquetebol, o valor médio da HDL-C elevou-se para um valor aceitável $(55,75$ $\mathrm{mg} / \mathrm{dL}$ ). Mesmo não exercendo um efeito protetor, estes novos valores não aumentam o risco de uma doença cardiovascular. Esta condição foi corroborada pela diminuição das razões LDL-C/HDL-C e CT/HDL-C observadas após a inserção na atividade, sendo similar ao ocorrido em um estudo prospectivo com intervenção de atividade física ${ }^{19}$.

A literatura vem demonstrando que um nível de atividade física regular mais elevado é capaz de alterar positivamente os níveis da HDL-C na população com LM, contudo, os aumentos da HDL-C observados no grupo PR através deste levantamento retrospectivo, foram de certa forma surpreendentes. Apesar da freqüência semanal na atividade, esses resultados mostram uma importante tendência do aumento da HDL-C concomitante a um aumento do gasto energético semanal através de atividade física desportiva, já que todos os pacientes não referiram nenhuma mudança importante no nível de atividade física diária, exceto pela prática do basquetebol. Isto nos leva a crer que uma atividade física mais freqüente, direcionadas a este perfil de pessoas, pode proporcionar resultados ainda mais representativos. De fato, alguns estudos envolvendo pessoas com LM mostraram aumentos na HDL-C após treinamento físico ${ }^{19,22}$, entretanto, estes 
resultados foram conseguidos através de sessões realizadas 3 a 4 vezes por semana .

Por outro lado, a inatividade física mostra-se gradualmente um hábito deletério aos níveis do HDL$\mathrm{C}^{9}$. Embora seja uma queda não significativa, a HDL-C mostrou uma tendência à queda conforme o tempo de inatividade após a LM. O valor médio da HDL-C do grupo NPR representa um risco aumentado de aproximadamente $12 \%$ em relação à população geral, baseado nas análises do estudo de Framingham ${ }^{6}$.

Ainda que os grupos tenham sido constituídos de forma homogênea (pacientes do sexo feminino, de meia idade, não fumantes, não etilistas, portadoras de lesões crônicas que utilizam preponderantemente a CR como auxílio locomoção), um importante fator, até pela própria característica do estudo, não foi possível controlar: "Qual é o nível de gasto energético que essas mulheres dispendem no dia-a-dia?". Será que a própria atividade de basquetebol não estimulou a adoção de um estilo de vida mais ativo? Provavelmente outros estudos serão necessários para avaliar o impacto de um programa de atividade física no cotidiano destas pessoas. Por ora, é útil considerar que é mais prático tornar-se mais ativo do que bem condicionado e que, reconhecidamente, ambas as condições diminuem o risco de morte.

Outra importante limitação diz respeito ao grupo NPR. Não sabemos exatamente o nível de atividade física destas pacientes, embora o relato, na imensa maioria das vezes, nas admissões e revisões no programa de reabilitação seja de total sedentarismo. Além disso, o número pequeno de indivíduos estudados no grupo PR reduz o poder de generalização dos nossos dados. Mesmos cientes desta dificuldade, optamos por uma análise estatística do tipo paramétrica, permitindo que os resultados deste estudo pudessem ser melhor comparados com estudo de análise e natureza semelhante ${ }^{19}$. Ademais, este estudo foi importante para o nosso "despertar" em relação ao problema, constituindo-se um ponto de partida para uma investigação que estamos conduzindo, com um número maior de participantes, visando contribuir para um maior aprofundamento do problema.

Portanto, o estudo não só sugere que a atividade física teve um grande potencial na elevação do HDLC nesta população como legitima a importância da atividade física na prevenção de doenças. É cada vez mais premente a viabilização de políticas públicas que promovam o acesso de populações especiais a programas de atividades físicas em condições que se aproximem às da população geral.

Por mais imprescindível que seja para um indivíduo com LM adotar um estilo de vida ativo e que isto esteja comprovado através de uma gama enorme de benefícios ${ }^{22}$, ainda não existe consenso na literatura quanto à recomendação dos exercícios mais seguros e eficazes, visando elevar o HDL-C destes indivíduos. Infelizmente, ainda não estão bem estabelecidos os conhecimentos capazes de sustentar diretrizes básicas, quanto ao tipo, a freqüência, a intensidade e a duração dos exercícios ${ }^{18}$. Assim, faz-se necessário um maior aprofundamento do problema, através de estudos experimentais de caráter longitudinal, a fim de verificar o efeito de diferentes exercícios que possam contribuir para a modificação do perfil de alto risco para doenças cardiovasculares, encontrado nas pessoas com LM, podendo assim proporcionar uma melhora na qualidade de vida e longevidade.

Também não podemos perder de vista a influência positiva que um programa de atividade física apropriado a esta população exerce sobre aspectos psicossociais como melhora da auto-estima, auto imagem, sociabilidade, independência, etc. O programa de atividade física proposto deve não só reconhecer o caráter indissociável destes aspectos, bem como criar condições que possam aprimorar ainda mais estas interações.

\section{CONCLUSÃO}

O estudo sugere que o BCR, como atividade física semanal, direta ou indiretamente, tem um grande potencial na elevação da HDL-C também em mulheres com LM, devido às mudanças significativas observadas neste estudo.

\section{REFERÊNCIAS BIBLIOGRÁFICAS}

1. De Lisa JA. Rehabilitation medicine: principles and practice. Philadelphia-USA: JB Lippincot; 1988.

2. Whiteneck GG, Charlifue SW, Frankel HL, Fraser MH, Gardner BP, Gerhart KA et al. Mortality, morbidity and psychosocial outcomes of persons spinal cord injured more than 20 years ago. Paraplegia 1992;30:617-30.

3. Yekutiel M, Brooks ME, Ohry A, Yarom J, Carel R. The prevalence of hypertension, ischaemic heart disease and diabetes in traumatic Spinal cord injuried patients and amputees. Paraplegia 1989;27:58-62.

4. Frankel H, Coll JR, Cahrlifue S, Whiteneck GG, Gardner BP, Jamous MA. et al. Long-term survival in spinal cord injury: a fifty year investigation. Spinal Cord 1998;36:266-74.

5. Stampfer MJ, Sacks FM, S Salvini, Willet WC, Hennekens $\mathrm{CH}$. A prospective study of cholesterol, apolipoproteins and the risk of myocardial infarction. $\mathrm{N}$ Engl $\mathrm{J}$ Med 1991;325(6):373-81.

6. Castelli WP, Garrison RJ, Wilson PWF, Abbot RD, Kalousdian S, Kannel WB. Incidence of coronary heart disease and lipoprotein cholesterol levels: the Framingham Study. JAMA 1986;256(20):2835-2838.

7. Mc Ardle WD, Katch FI, Katch VL. Fisiologia do exercício: energia, nutrição e desempenho humano. Rio de janeiro: Ed. Guanabara Koogan; 1998.

8. Dioguardi GS, Faludi AA, Bertolami MC. Lípides nas atividades Física e desportiva. In: Ghorayeb N, Barros Neto TL, editores. O Exercício. São Paulo: Ed. Atheneu, 1999; p. 107-16.

9. Gorgatti MG, Bohme MTS. Atividade Física e Lesão Medular. In: Gorgatti MG, Costa RF. Editores, Atividade física Adaptada. Barueri: Ed. Manole, 2005; p. 147-81.

10. Walker J, Shepard R. Cardiac Risk factors immediately follow spinal injury. Arch Phys Med Rehabil 1993;74:112933.

11. Bauman W, Spungen AM. Disorders of carbohydrate in lipid metabolism in veterans with paraplegia or quadriplegia: a model of premature aging. Metabolism 1994;43(6):749-55. 
12. Demirel S, Demirel G, Turek T, Erk O, Yilmaz G. Risk factors for coronary disease in patients with spinal cord injury in Turkey. Spinal Cord 2001;39:134-38.

13. Lammertse DP. Maintaining health long-term with spinal cord injury. Top Spinal Cord Inj Rehabil 2001;6(3):1-21.

14. Tran ZV, Weltman A, Glass GV, Mood DP. The effects of exercise on blood lipids and lipoproteins: a meta-analysis of studies. Med Scie Sports Exerc 1983;15(5):393-02.

15. Prado ES, Dantas EHM. Efeitos dos exercícios físicos aeróbio e de força nas lipoproteínas HDL, LDL e lipoproteína (a). Arq Bras Cardiol 2002;79(4):429-33.

16. Cambri LT, Souza M, Mannrich G, Cruz RO, Gevaerd MS. Perfil lipídico, dislipidemias e exercícios físicos. Rev Bras Cineantropom Desempenho Hum 2006;8(3):100-06.

17 .Brenes G, Dearwater S, Shapera R. High density lipoprotein cholesterol concentrations in physically active and sedentary spinal cord injured patients. Arch Phys Med Rehabil 1986;67:445-50.

18. Washburn RA, Figoni SF. Physical activity and chronical cardiovascular disease prevention in spinal cord injury: a comprehensive literature review. Top Spinal Cord Inj Rehabil 1998;3(3):16-32.

19. Nash MS, Jacobs PL, Mendez AJ, Golberg RB. Circuit resistance training improves the atherogenic lipid profiles of persons with chronic paraplegia. J Spinal Cord Med 2000;24(1):2-9.
20. Sociedade Brasileira de Cardiologia. III Diretrizes Brasileiras sobre Dislipidemias e Diretriz de Prevenção da Aterosclerose do Departamento de Aterosclerose. Arq Bras Cardiol 2001;77(sup 3):1-48.

21. Storch MJ, Konig D, Bultermann D, Blum A, Vogt S, Baumstark $M$ et al. Lipid profile in spinal cord injured women with different levels. Preventive Medicine 2005;40:321-25.

22. Sorg RS. HDL-Cholesterol: Exercise Formula. Results of long-term (6 years) strenuous swimming exercise in a middle-age male with paraplegia. JOSPT 1993;17:195-99.

22. Lacourse MG, Lawrence KE, Cohen MG, Young RK. Lesão da Medula espinhal. In: Frontera RW, Dawson DM, Slovik DM, Editores. Exercício físico e reabilitação. Porto Alegre: Artmed Editora; 2001; 246-67.

\section{Agradecimentos}

A Cristiano Barreira da Ponte por todo apoio na confecção e organização da parte gráfica do artigo, ao Dr. Enio Comerlato pelas sugestões e críticas e ao Dr. Daniel de Paula Lima e Oliveira Lopes pelas sugestões, críticas e incentivo.

\section{Endereço para correspondência}

Fabio Barreto Rodrigues

Rua Lídia Brigido, 367 apto 03, Parque Manibura

Recebido em 17/04/07

Fortaleza-CE, Brasil.

E-mail: fabiobarreto@sarah.br 